\title{
Gestión de tesorería en las empresas contratistas para puertos
}

\author{
Treasury management in port contractor companies
}

\section{Gestão de tesouraria em empreiteiras portuárias}

Recibido: mayo 2019

Arbitrado: junio 2019

Publicado: septiembre 2019

\author{
《 Osnely Castellano \\ osnelycastellano@hotmail.com \\ ORCID: 0000-0002-4001-2263
}

Universidad del Zulia, Venezuela

\section{RESUMO}

\section{RESUMEN}

El objetivo se centró en analizar la gestión de tesorería utilizada por las empresas contratistas del Puerto de Maracaibo, Venezuela. La metodología fue descriptiva, con diseño no experimental, de campo y transeccional. La población quedó conformada por las siete (7) empresas con más cantidad de contratos al momento de la investigación. La información se recolecto a través de un cuestionario de 10 ítems, con escala de frecuencias. El mismo fue validado por el juicio de expertos y la confiabilidad fue calculada por la formula Alfa de Cronbach, obteniéndose 0,98. Para procesar los resultados se empleó la media aritmética $(X)$ y la frecuencia relativa. Se evidencio una moderada utilización de la gestión de tesorería como unidad estratégica que debe manejar en forma efectiva y eficiente los recursos financieros de las áreas financieras analizadas.

Palabras clave: Áreas financieras; gestión de tesorería; puerto de Maracaibo; recursos financieros, unidad estratégica
The objective was focused on analyzing the treasury management used by the contracting companies of the Port of Maracaibo, Venezuela. The methodology was descriptive, with a non-experimental, field and transectional design. The population was made up of the seven (7) companies with the highest number of contracts at the time of the investigation. The information was collected through a 10-item questionnaire, with a frequency scale. It was validated by the expert judgment and the reliability was calculated by the Cronbach's Alpha formula, obtaining 0.98 . To process the results, the arithmetic mean $(X)$ and the relative frequency were used. There was evidence of a moderate use of treasury management as a strategic unit that must effectively and efficiently manage the financial resources of the financial areas analyzed.

Key words: Financial areas; treasury management; port of Maracaibo; financial resources, strategic unit
O objetivo foi analisar a gestão de tesouraria utilizada pelas empresas contratantes do Porto de Maracaibo, Venezuela. A metodologia foi descritiva, com desenho não experimental, de campo e transversal. A população foi constituída pelas sete (7) empresas com maior número de contratos à época da investigação. As informações foram coletadas por meio de questionário de 10 itens, com escala de frequência. Foi validado pelo julgamento de especialistas e a confiabilidade calculada pela fórmula do Alfa de Cronbach, obtendo-se 0,98. Para processar os resultados, foram utilizadas a média aritmética $(X)$ e a frequência relativa. Evidenciou-se uma utilização moderada da gestão de tesouraria como unidade estratégica que deve gerir de forma eficaz e eficiente os recursos financeiros das áreas financeiras analisadas.

Palavras-chave: Áreas financeiras; gestão de tesouraria; porto de Maracaibo; recursos financeiros, unidade estratégica 


\section{INTRODUCCIÓN}

$\mathrm{L}$ os puertos como parte del transporte marítimo, demandan adaptación a las nuevas realidades y necesidades financieras, para responder con eficiencia las consecuencias de la globalización. Bajo la economía de mercado, los puertos tienen una gran presión de la sociedad privada y de los gobiernos, teniendo que prosperar en tales condiciones, por lo cual requieren de herramientas para dar respuesta rápida y acertada; una de las herramientas de uso común es la gestión financiera (Barrientos, 2009).

Por ello, los puertos marítimos los cuales son el contexto de esta investigación, hacen referencia a la búsqueda del equilibrio entre los recursos disponibles y los objetivos fijados, a través de la optimización de los mismos. En este sentido corresponde a disciplinas tales como el control de gestión, gestión de inversión, gestión de tesorería, de la mano con el cumplimiento de las normativas legales propias de cada país, centrar sus actividades en el dominio del trabajo actual y su objetivo es principalmente la eficiencia.

Al respecto, para la Comisión Económica para América Latina y el Caribe (CEPAL) (2006), la gestión portuaria a nivel financiero debe solventar deficiencias en cuanto al establecimiento de líneas generales definiendo lo que hay que hacer y los métodos a utilizar para lograr los objetivos propuestos.

En otras palabras, es pensar en el futuro para tomar decisiones y hacerlas operativas como órdenes, instrucciones y reglas, agregándolas en el establecimiento de vínculos de integración entre las distintas partes del todo, con un esfuerzo de cooperación facilitando el funcionamiento y el éxito asegurando la armonía. Esto permite reconocer una dificultad financiera, cuyos signos pueden ser proporcionados tanto por las fuentes internas como externas de la empresa; entre algunos signos se pueden mencionar la disminución de los ingresos, el aumento de las necesidades de capital circulante, el aumento de los gastos financieros, entre otros.

Cabe destacar, a este nivel del discurso, en cuanto a la gestión de tesorería de puertos marítimos, que en Venezuela, país de América Latina, se suscriben convenios comerciales con el resto del mundo, manteniendo un tránsito marítimo de relevancia para el acontecer socioeconómico interno y externo. Uno de sus puertos marítimos más destacados es el de Maracaibo por el intercambio de mercancía con los países andinos, caribeños, el océano Atlántico, el Canal de Panamá, sur de los Estados Unidos y Centroamérica; esto incluye la transferencia, carga, descarga y realización de servicios a buques o servicios a la carga de los mismos, específicamente ofrece servicios para la carga refrigerada (Montero, 2007).

Este puerto conviene negocios con diferentes empresas contratistas tales como Mecanizado Industrial y Mantenimiento, Grating, Comercializadora e Inversiones OSNYCA, Corporación Toncan Digital, Proveedores y Servicios Venezuela, PC Suministros 2000, Grupo Firmo, entre otras, cuyas 
actividades económicas ponen al servicio del puerto incluyendo mantenimiento de equipos e instalaciones, alquiler de equipos de manejo de cargamento y más.

Dichas empresas contratistas, mantienen relación comercial con la administración del Puerto de Maracaibo en Venezuela bajo la figura de Contrataciones Públicas, para realizar servicios específicos, concerniente a los almacenes, sitios, patios, ubicados en el área primaria. En este sentido, el proceso de contratación pública implica un concurso público manejado por la administración del puerto de Maracaibo donde la empresa ganadora pasa a ser responsable del cumplimiento del contrato adquirido.

Por lo tanto, permitió destacar como una buena gestión de tesorería de las empresas portuarias no evalúa solamente si se dispone o no de dinero en el presente; se trata de planificar, de prever una buena gestión a futuro con las probables faltas o excesos de dinero. Sin embargo, en los puertos estas son variables de difícil control y previsión. Dentro de este marco, plantea Montero (2007), "la meta final será siempre incrementar la calidad y eficiencia de los servicios y reducir los costes, o lo que es lo mismo, satisfacer las necesidades del cliente usuario del puerto" (p. 23).

Ahora bien, se considera que estas empresas se deben posicionar en el universo del mercado de capitales y el de los negocios, siendo estos los medios o el contexto financiero donde se maneja su capital. Dichos recursos deben ser gestionados de manera alineada con la actividad económica, bajo controles y procesos de retroalimentación que permitan prestar servicios de calidad bajo el mejor aprovechamiento y eficiencia.

En este sentido, la organización a nivel de gestión de tesorería en las contratistas debe buscar satisfacer las necesidades de su cliente, como lo es el Puerto de Maracaibo, en términos de rentabilidad, estableciendo relaciones de ganar-ganar, lo que lleva a la actuación estratégica en cuanto al análisis de las actividades, rentabilidad, capital invertido y la financiación para medir su rendimiento.

\section{MÉTODO}

$\mathrm{L}$ a metodología fue descriptiva, con diseño no experimental, de campo y transeccional. Arias (2004) señala que estos estudios consisten en la caracterización de un hecho, fenómeno o grupo con el fin de establecer su estructura o comportamiento, midiendo de forma independiente la variable; es decir, este tipo de estudio describe los acontecimientos sin realizar ninguna inferencia, ni manipularlos.

La población quedó conformada por las siete (7) empresas con más cantidad de contratos al momento de la investigación. La información se recolecto a través de un cuestionario de 10 ítems, con escala de frecuencias. El mismo fue validado por el juicio de expertos y la confiabilidad fue calculada por la formula Alfa de Cronbach, obteniéndose 0,98. 
El tratamiento estadístico se concretó a través de las medias ponderadas, de acuerdo al cuadro 1, esto permitió estudiar los datos con sistematización lógica y explicativa. Complementariamente, se tomó que la suma de las frecuencias relativas de las alternativas (siempre y casi siempre) para los indicadores cuando supera el $75 \%$ se considera como tendencia positiva la gestión financiera, tal como se aprecia en el cuadro 2.

Cuadro 1. Baremo para la interpretación de la media aritmética.

\begin{tabular}{cccc}
\hline Alternativas & Opción de respuesta & Rango & Nivel de respuesta \\
\hline $\mathbf{5}$ & Siempre(S) & $4.20 \leq \bar{\chi} \leq 5.00$ & Muy alta utilización \\
$\mathbf{4}$ & Casi Siempre (CS) & $3.40 \leq \bar{\chi}<4.20$ & Alta utilización \\
$\mathbf{3}$ & Algunas Veces (AV) & $2.60 \leq \bar{\chi}<3.40$ & Moderada utilización \\
$\mathbf{2}$ & Casi Nunca (CN) & $1.80 \leq \bar{\chi}<2.60$ & Baja utilización \\
$\mathbf{1}$ & Nunca(N) & $1.00 \leq \bar{\chi}<1.80$ & Muy baja utilización. \\
\hline
\end{tabular}

Cuadro 2. Categoría de análisis para la frecuencia relativa.

\begin{tabular}{cc}
\hline Alternativa & Tendencia \\
\hline S + CS $\geq 75 \%$ & La tendencia se considera positiva \\
$\mathbf{4 5 \%} \leq \mathbf{S}+\mathbf{C S}<\mathbf{7 5 \%}$ & La tendencia se considera neutral \\
$\mathbf{S}+\mathbf{C S}<\mathbf{4 5 \%}$ & La tendencia se considera negativa \\
\hline
\end{tabular}

\section{RESULTADOS Y DISCUSIÓN}

omo se puede apreciar en la tabla 1, se presenta el resumen para la gestión de tesorería, cuya tendencia fue negativa $(42,86 \%)$ con un nivel de respuesta de moderada utilización de la gestión de tesorería en las áreas financieras analizadas, dada la baja utilización de los pagos a plazos seguida de la moderada utilización de pagos de contado, alta utilización de políticas de créditos y muy alta utilización de procedimientos de cobro. 
Tabla 1. Gestión de tesorería.

\begin{tabular}{|c|c|c|c|c|c|c|}
\hline Indicador & $\bar{\chi}$ & $\begin{array}{l}\text { Nivel de } \\
\text { Respuesta }\end{array}$ & $\bar{\chi}$ & $\begin{array}{c}\text { Nivel de } \\
\text { Respuesta }\end{array}$ & FR (S+CS) & Tendencia \\
\hline $\begin{array}{l}\text { Políticas de } \\
\text { Cobranza }\end{array}$ & 2,98 & $\begin{array}{l}\text { Moderada } \\
\text { utilización }\end{array}$ & & & & \\
\hline $\begin{array}{l}\text { Procedimientos de } \\
\text { cobro }\end{array}$ & 4,79 & $\begin{array}{l}\text { Muy alta } \\
\text { utilización }\end{array}$ & & & & \\
\hline Políticas de Crédito & 3,62 & Alta utilización & 3,24 & $\begin{array}{l}\text { Moderada } \\
\text { utilización }\end{array}$ & $42,86 \%$ & Negativa \\
\hline De Contado & 2,88 & $\begin{array}{l}\text { Moderada } \\
\text { utilización }\end{array}$ & & & & \\
\hline A Plazos & 1,95 & Baja utilización & & & & \\
\hline
\end{tabular}

Las afirmaciones anteriores son validadas parcialmente en los postulados de Piñeiro et al (2006) quienes señalan que una amplia mayoría de las operaciones de las empresas implica, antes o después, un movimiento de tesorería. Estas operaciones son críticas para su actividad y pueden causar costes de oportunidad así como gastos financieros explícitos por transacción, lo que establece un claro nexo con el objetivo de maximización de la riqueza de los propietarios, en tal sentido, el objetivo de gestión consiste en minimizar el saldo de tesorería, al tiempo que se garantiza la disponibilidad de los fondos precisos para afrontar los pagos corrientes.

De acuerdo con estos resultados, se señaló, quienes gestionan estas empresas deben utilizar los instrumentos adecuados de financiamiento para mejorar su gestión de tesorería, por cuanto esta representa un área financiera importante donde se negocian las mejores condiciones con las entidades financieras.

Al detalle en la tabla 2, se logra observar en relación al indicador políticas de cobranzas, que existe una tendencia negativa (26,19\%) concordante con el nivel de respuesta de moderada utilización $(2,98)$ producto de las respuestas a los ítems 1 y 2 develando moderada utilización $(2,38)$ en el cumplimiento de la política de cobranza con los niveles de recaudación esperados y alta utilización $(3,57)$ en el empleo de la gestión de cobro como política financiera. 
Tabla 2. Políticas de Cobranza.

\begin{tabular}{|c|c|c|c|c|c|c|}
\hline Ítem & $\bar{\chi}$ & $\begin{array}{c}\text { Nivel de } \\
\text { Respuesta }\end{array}$ & $\bar{\chi}$ & $\begin{array}{c}\text { Nivel de } \\
\text { Respuesta }\end{array}$ & $F R(S+C S)$ & Tendencia \\
\hline $\begin{array}{l}\text { 1. Cumplen la política } \\
\text { de cobranza con los } \\
\text { niveles } \\
\text { recaudación } \\
\text { esperados }\end{array}$ & 2,38 & $\begin{array}{l}\text { Moderada } \\
\text { utilización }\end{array}$ & 2,98 & $\begin{array}{l}\text { Moderada } \\
\text { utilización }\end{array}$ & $26,19 \%$ & Negativa \\
\hline $\begin{array}{l}\text { 2. Emplean la gestión de } \\
\text { cobro como política } \\
\text { financiera }\end{array}$ & 3,57 & Alta utilización & & & & \\
\hline
\end{tabular}

Los resultados evidencian de forma parcial los postulados de Escribano (2011), en cuanto a que una efectiva labor de cobranza está relacionada directamente con una efectiva una política de créditos, por lo que se minimiza los gastos de cobro por cuentas difíciles o de dudosa recuperación.

De igual manera, los datos antes descritos dan evidencia que en las áreas financieras analizadas existe una moderada utilización de las políticas de cobranza aspecto que reviste importancia para el desarrollo de una buena gestión de tesorería para optimizar los recursos monetarios.

Seguidamente, se presentan en la tabla 3, los resultados para el indicador procedimientos de cobro, en el que se observa una tendencia de respuestas positiva $(95,24 \%)$ congruentes con el nivel de respuesta muy alta utilización $(4,29)$ de los procedimientos, en razón de una muy alta utilización $(4,90)$ de los procedimientos de cobro direccionados hacia el cumplimiento de la gestión financiera esperada y también los procedimientos de cobro anticipados y planificados deliberadamente de modo que sigan una serie de pasos o etapas en forma regular y ordenada $(4,67)$.

Los resultados expuestos ratifican los principios teóricos de Olsina (2009), quien señala que deben ser anticipados y planificados deliberadamente de modo que sigan una serie de pasos o etapas en forma regular y ordenada. 
Tabla 3. Procedimientos de cobro.

\begin{tabular}{|c|c|c|c|c|c|c|}
\hline Ítem & $\bar{\chi}$ & $\begin{array}{c}\text { Nivel de } \\
\text { Respuesta }\end{array}$ & $\bar{\chi}$ & $\begin{array}{c}\text { Nivel de } \\
\text { Respuesta }\end{array}$ & $F R(S+C S)$ & Tendencia \\
\hline $\begin{array}{l}\text { 3. Emplean los } \\
\text { procedimientos de } \\
\text { cobro direccionados } \\
\text { hacia el cumplimiento } \\
\text { de la gestión } \\
\text { financiera esperada }\end{array}$ & 4,90 & $\begin{array}{l}\text { Muy alta } \\
\text { utilización }\end{array}$ & & & & \\
\hline $\begin{array}{l}\text { 4. Utilizan los } \\
\text { procedimientos de } \\
\text { cobro anticipados y } \\
\text { planificados } \\
\text { deliberadamente de } \\
\text { modo que sigan una } \\
\text { serie de pasos o } \\
\text { etapas en forma } \\
\text { regular y ordenada }\end{array}$ & 4,67 & $\begin{array}{l}\text { Muy alta } \\
\text { utilización }\end{array}$ & 4,79 & $\begin{array}{l}\text { Muy alta } \\
\text { utilización }\end{array}$ & $95,24 \%$ & Positiva \\
\hline
\end{tabular}

Po su parte, ante los resultados positivos en cuanto a la muy alta utilización de los procedimientos de cobro, se consideró una fortaleza para la maximización del cobro y minimización de las pérdidas de cuentas por cobrar.

En el mismo orden de ideas, en la tabla 4 se puede apreciar los resultados para el indicador políticas de crédito, mostrando una tendencia neutral $(47,62 \%)$ y alta utilización $(3,62)$ refiriendo los encuestados alta utilización $(3,67)$ que se explican los lineamientos técnicos que utiliza el gerente financiero de la empresa, con la finalidad de otorgar facilidades de pago a un determinado cliente, de igual manera consideran alta utilización $(3,57)$ que la ejecución de las políticas de crédito permiten alcanzar los objetivos financieros trazados.

Así que, la alta utilización de las políticas de crédito refiere una condición saludable para la gestión de tesorería en las empresas contratistas del puerto de Maracaibo, dándole fortaleza, lo cual pone de manifiesto los postulados de Ortíz (2005), al señalar que la empresa no debe solo ocuparse de los estándares de crédito que establece, sino también de la utilización correcta de estos estándares al tomar decisiones de crédito, para lo cual deben desarrollarse fuentes adecuadas de información y métodos de análisis de crédito. 
Tabla 4. Políticas de Crédito.

\begin{tabular}{lcccccc}
\hline Ítem & $\bar{\chi}$ & $\begin{array}{c}\text { Nivel de } \\
\text { Respuesta }\end{array}$ & $\bar{\chi}$ & $\begin{array}{c}\text { Nivel de } \\
\text { Respuesta }\end{array}$ & FR (S+CS) & Tendencia \\
\hline $\begin{array}{l}\text { 5. Se explican los lineamientos } \\
\text { técnicos que utiliza el }\end{array}$ & 3,67 & $\begin{array}{c}\text { Alta } \\
\text { utilización }\end{array}$ & & & \\
gerente financiero de la \\
empresa, con la finalidad \\
de otorgar facilidades de \\
pago a un determinado \\
cliente
\end{tabular}

En este recorrido de resultados, se presentan en la tabla 5 el comportamiento obtenido para el indicador pago de contado, en ella se observa una tendencia neutral $(45,24 \%)$ con un nivel de respuesta de moderada utilización $(2,88)$ de la gestión de tesorería de contado, ya que los encuestados, consideran que los clientes cumplen su pago al contado en la forma acordada $(4,67)$; no obstante se presenta una muy baja utilización $(1,10)$ al considerar que se emplea el pago de contado para compras de mucho valor.

En consideración, la moderada utilización del pago de contado ofrece una oportunidad de mejora en la gestión de tesorería, validando de cierta forma los resultados, lo referido por Escribano (2011), quien señala que el pago al contado es una voz comercial que equivale en efectivo, en dinero, en especie y significa la forma de pago sin plazo señalado, o sea, al momento de contraerse la deuda, originada por la entrega de mercancías, en contraposición a la forma de pago a plazos. 
Tabla 5. Pago de contado.

\begin{tabular}{|c|c|c|c|c|c|c|}
\hline Ítem & $\bar{\chi}$ & $\begin{array}{c}\text { Nivel de } \\
\text { Respuesta }\end{array}$ & $\bar{\chi}$ & $\begin{array}{c}\text { Nivel de } \\
\text { Respuesta }\end{array}$ & FR $(S+C S)$ & Tendencia \\
\hline $\begin{array}{l}\text { 7. Consideran que los } \\
\text { clientes cumplen su } \\
\text { pago al contado en la } \\
\text { forma acordada }\end{array}$ & 4,67 & $\begin{array}{l}\text { Muy alta } \\
\text { utilización }\end{array}$ & 2,88 & $\begin{array}{l}\text { Moderada } \\
\text { utilización }\end{array}$ & $45,24 \%$ & Neutral \\
\hline $\begin{array}{l}\text { 8. Emplean el pago de } \\
\text { contado para compras } \\
\text { de mucho valor }\end{array}$ & 1,10 & $\begin{array}{l}\text { Muy baja } \\
\text { utilización }\end{array}$ & & & & \\
\hline
\end{tabular}

Asimismo, la tabla 6 recoge el comportamiento obtenido para el indicador pago a plazos que obtuvo una tendencia negativa $(0,00 \%)$ concordante con la baja utilización $(1,95)$ que arroja el nivel de respuestas con respecto al pago a plazos en las áreas financieras estudiadas, en razón de la muy baja utilización $(1,52)$ en la adquisición de bienes duraderos comprados a plazos y en el uso de los distintos lapsos de tiempo para hacer frente a una serie de pagos $(2,38)$.

Tabla 6. Pago a plazos.

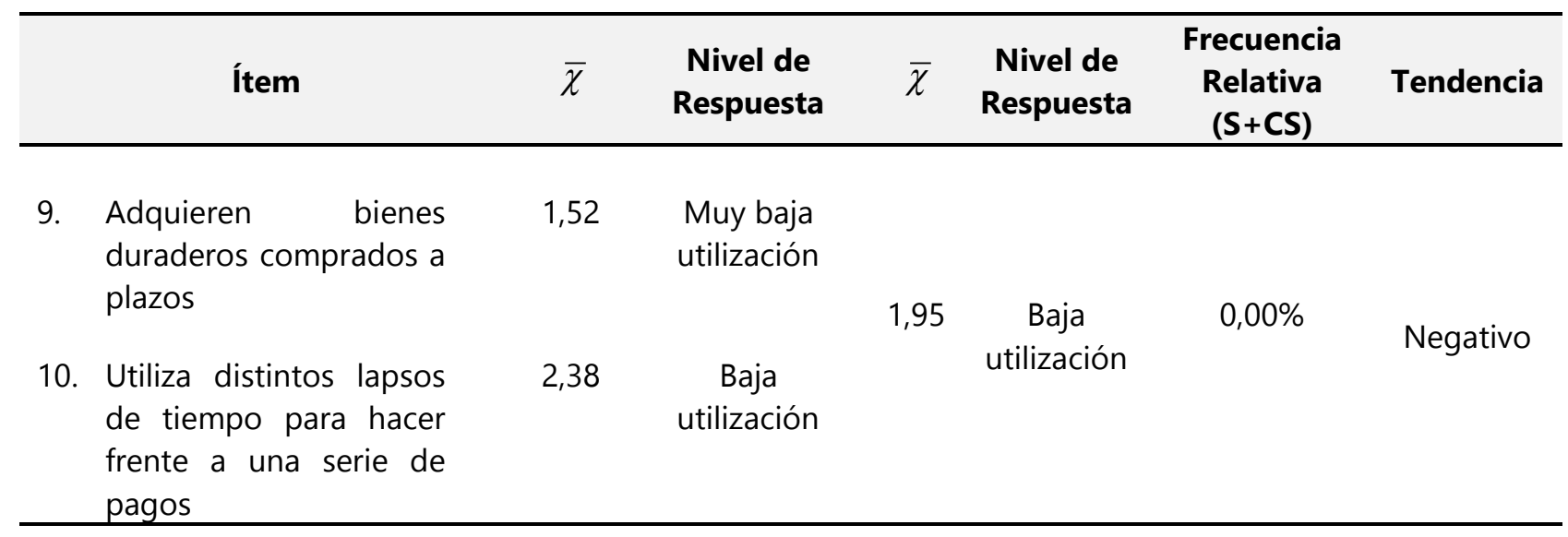

Por lo tanto, los pagos a plazos revisten una debilidad para la gestión de tesorería, por tanto los resultados presentados no validan los postulados de Escribano (2011), quien considera que el pago a plazos es un procedimiento compuesto de un crédito en donde el comprador paga por un producto al hacer una serie de pagos en distintos lapsos de tiempo. Para el citado autor se 
denomina pago a plazos a una modalidad utilizada normalmente para bienes duraderos, a través del cual el pago del precio no se hace en el momento de la adquisición del bien, sino que se difiere en el tiempo a través de una serie de pagos denominados plazos, cuotas, abonos o letras.

Las afirmaciones anteriores, de moderada utilización de la gestión de tesorería, le permite inferir que, a las gerencias de las empresas analizadas se le presentan grandes retos para operar responsablemente esos recursos, por cuanto el gerente está rodeado de constantes cambios en el entorno, a veces difíciles de sortear, lo que demandan la implementación de estrategias que sirvan de base para prever o atenuar el impacto de esos cambios en dichas empresas.

\section{CONCLUSIONES}

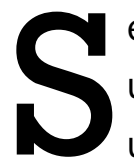
e revela una tendencia fue negativa $(42,86 \%)$, con un nivel de respuesta de moderada utilización de la gestión de tesorería en las áreas financieras analizadas, dada la baja utilización de los pagos a plazos seguida de la moderada utilización de pagos de contado, alta utilización de políticas de créditos y muy alta utilización de procedimientos de cobro.

Es importante mencionar que la gestión de tesorería representa un área financiera importante dentro de las empresas contratistas estudiadas desde donde se negocian las mejores condiciones con las entidades financieras, o bien utilizando los instrumentos adecuados de financiamiento por cuanto su moderada utilización refleja una debilidad.

\section{REFERENCIAS}

Barrientos, M. (2009). Manual de gestión financiera. Disponible

en: http://www.hacienda.go.cr/cifh/sidovih/cursos/ material_de_apoyo-F-C-

$\mathrm{CIFH} / 1$ MaterialdeapoyocursosCIFH/12Programa FormacRectoriaFinanciera/ManualGestionFinanc .pdf

Comisión Económica para América Latina y el Caribe (Cepal). (2006). Indicadores de productividad para la industria portuaria. Aplicación en América Latina y el Caribe

Escribano, G. (2011). Las finanzas corporativas. Paraninfo. Madrid. España.
Montero, L. (2007). Evolución de los sistemas de gestión de los puertos. Disponible en: http://www.aippyc.org/materiales_archivos/Mod elos-Gestion-Portuaria-Montero.pdf

Olsina, F (2009). Etión de tesorería. Optimizando los flujos monetarios. Profit Editorial Barcelona, España

Ortíz, A. (2005). Gerencia financiera y diagnostico financiero. Mc Graw Hill. Colombia.

Piñeiro, C., De llano P., Álvarez B., (2006). Dirección Financiera: modelos avanzados de decisión. La Coruña. España. Delta Publicaciones. 